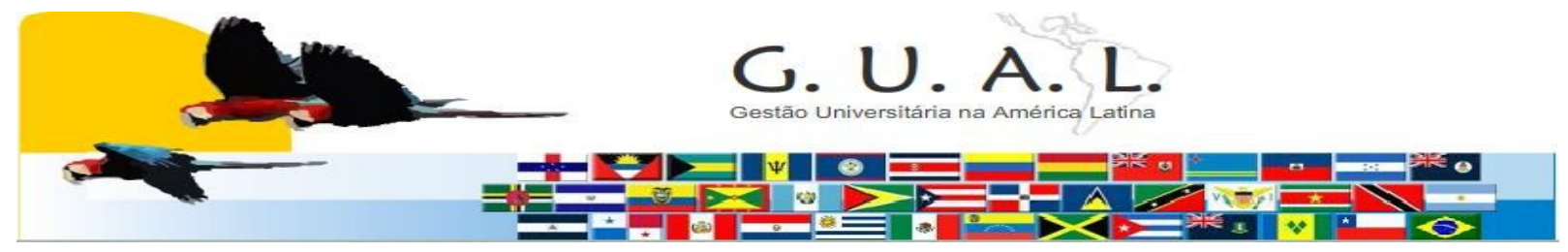

ISSN 1983-4535

\title{
PROGRAMAS DE POSGRADO DE FINANCIAMIENTO COMPLEMENTARIO: MITOS Y REALIDADES
}

\author{
Gabriela Marín Raventós, Doctora \\ Universidad de Costa Rica \\ gabriela.marin@ucr.ac.cr
}

\begin{abstract}
RESUMO
Se trata de presentar los mitos y realidades de lo que se ha denominado la privatización de la educación superior universitaria en el contexto de una universidad pública. Se presenta una comparación entre programas de posgrado totalmente financiados con fondos públicos, programas regulares, y programas de posgrado de financiamiento complementario. El estudio se basa en los datos auto-reportados por los programas en su Informe de Labores del 2010 realizado con indicadores de gestión del Manual de Acreditación de la Agencia Centroamericana de Acreditación de Postgrado, ACAP, y elaborado por el Sistema de Estudios de Posgrado de la Universidad de Costa Rica utilizando una plataforma de software libre para realizar encuestas en línea. Se presentan las diferencias entre los programas regulares, los programas de financiamiento complementario y los mixtos, poniendo en evidencia los beneficios de este último tipo de gestión.
\end{abstract}

Palabras-clave: Gestión universitaria. Posgrados. Financiamiento. 
Costa Rica, desde que declara la abolición del ejército en 1948, ha invertido en educación y seguridad social, lo cual le permite gozar de un alto índice de desarrollo humano, el sexto mejor de Latinoamérica y el segundo en Centroamérica [9]. Mucho de su alto desarrollo humano es consecuencia de la calidad de su Educación Superior.

Con más de 70 años de existencia, la Universidad de Costa Rica (UCR) fue pionera en nuestro país. Posterior a ella, se crean tres universidades estatales más para responder a las necesidades del país: la Universidad Nacional (UNA), el Instituto Tecnológico de Costa Rica (ITCR), y la Universidad Estatal a Distancia (UNED). Estas universidades públicas nacen con la totalidad de su financiamiento garantizado por la Constitución de la República. Sin embargo, la crisis de los 80 s produce que el financiamiento estatal a estas universidades se estanque y que ellas deban buscar medios alternativos para su funcionamiento. A partir de este momento, la matrícula estudiantil crece con un ritmo menor al de la población, dejando una brecha en la capacidad de respuesta de la Educación Superior a las demandas del país.

En consecuencia, a partir de 1986, empiezan a proliferar las universidades privadas. Pasan de seis en ese primer año a cerca de 50 en el 2000. El crecimiento acelerado de los primeros años de privatización de la educación superior ha disminuido y para el 2010 existen 51 privadas que acompañan a las universidades públicas.

Hoy en día, las universidades del Estado absorben menos del 50\% de los estudiantes de secundaria que cada año solicitan ingresar al sistema. Los y las estudiantes compiten a través de exámenes de admisión para ingresar a las tres más antiguas, UCR, ITCR, y UNA. La UNED, universidad a distancia, es de libre acceso.

Las universidades privadas son heterogéneas en cuanto a su tamaño, áreas de especialización y calidad. Existen 2 o 3 de ellas que comienzan a ser competitivas con respecto de las universidades públicas.

En el presente artículo se trata de presentar los mitos y realidades de lo que se ha denominado la privatización de la educación superior universitaria en el contexto de una universidad pública.

\section{FINANCIAMIENTO DE LA EDUCACIÓN SUPERIOR ESTATAL}

El artículo 85 de la Constitución Política es el pilar en el que se fundamenta el financiamiento de la Educación Superior Universitaria Estatal costarricense, al expresar:

Rev. GUAL., Florianópolis, v. 4, n. 3, p.01-20, set/dez. 2011 
"El Estado dotará de patrimonio propio a la Universidad de Costa Rica, al Instituto Tecnológico de Costa Rica, a la Universidad Nacional y a la Universidad Estatal a Distancia y les creará rentas propias, independientemente de las originadas en estas instituciones. Además mantendrá -con las rentas actuales y con otras que sean necesarias un fondo especial para el financiamiento de la Educación Superior Estatal. (...)" [2]

Con el propósito de cumplir con lo estipulado en el artículo 85 de la Constitución Política de la República, el Consejo Nacional de Rectores (CONARE) propuso un mecanismo de reajuste automático de financiamiento, el cual se plasmó mediante una fórmula que buscaba consolidar el Fondo Especial para el Financiamiento de la Educación Superior (FEES). [3]

La propuesta fue aceptada por el Poder Ejecutivo y el "Convenio de Financiamiento de la Educación Superior" fue aprobado por la Comisión de Enlace en la Sesión N111, celebrada el 8 de noviembre de 1988 [3] . A partir de ese año, el gobierno y el CONARE negocian los términos del nuevo mecanismo de financiamiento para la Educación Superior cada quinquenio.

Los elementos fundamentales de estos convenios han sido:

- el mecanismo de reajuste automático del FEES,

- los recursos adicionales para proyectos específicos, y

- el compromiso de las universidades de generar ingresos propios.

En la sección inferior de la Figura 1 podemos observar la evolución de la Educación Superior en una línea de tiempo. Nótese que las Universidades Privadas comienzan a existir en el contexto de la crisis mundial de los 80 s y producto de los vacíos en financiamiento de la educación pública producto de las presiones de los organismos internacionales, tales como el Fondo Monetario Internacional, los cuales abogaban por la privatización parcial de la educación.

Como plantea María Pérez Yglesias, al referirse a la necesidad de las universidades públicas de buscar fuentes alternativas de financiamiento y no depender únicamente del FEES:

"Esta dicotomía se presenta como una clara consecuencia de las discusiones y los cambios en torno a la función del estado nacional y la tendencia a la liberalización y privatización en todos los niveles de acuerdo con patrones internacionales. En la Universidad de Costa Rica se discute sobre la actitud 
que debe asumir la institución frente a la eclosión de universidades privadas e, internamente, se polemiza sobre si la venta de servicios implica privatizar parcialmente o crear una "universidad paralela"... “ $[8$, pg. 97]

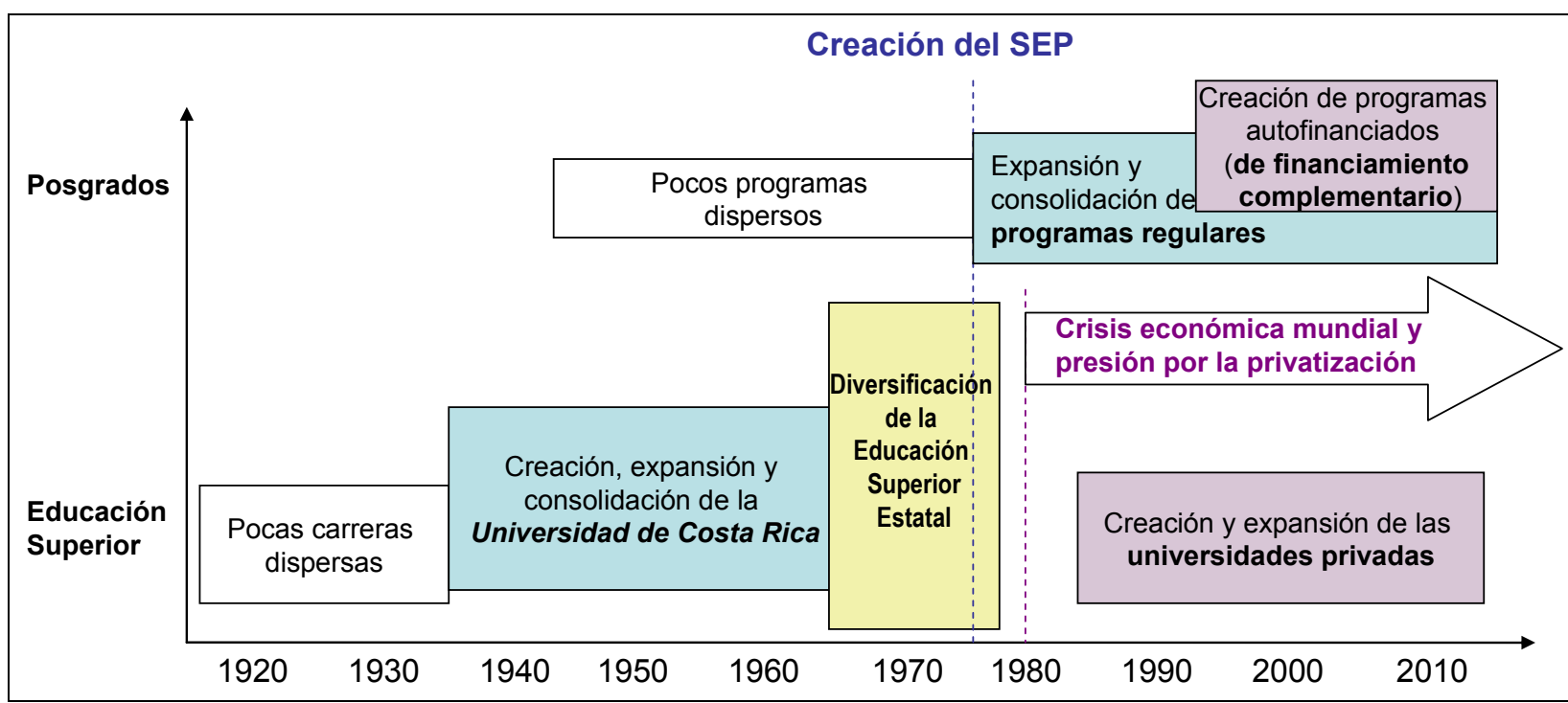

Figura 1 Evolución de la Educación Superior y de los Posgrados en la Universidad de Costa Rica en el contexto de la crisis mundial de los 80 s (elaboración propia).

En la anterior figura se puede observar como los posgrados en la Universidad de Costa Rica, al menos, unos pocos de ellos, nacen casi desde su creación en los años 40s. El Sistema de Estudios de Posgrado (SEP), como tal, inicia sus actividades en 1975 con tres programas en marcha: Microbiología, Filosofía y Ciencias Agrícolas y uno nuevo: Química. Inicia formalmente por mandato del Consejo Universitario, solo dos años después de la celebración del III Congreso Universitario donde se propone su creación como Sistema y no como una Escuela de Graduados independiente, como se ideó en la década anterior. Se concibe como un sistema integrador de esfuerzos distribuidos a lo largo de todas las unidades académicas, fuesen estas Escuelas, Facultades o Centros de Investigación.

Nace así una propuesta organizacional del posgrado en la Universidad de Costa Rica con naturaleza propia, que integra los programas ya existentes y que multiplica sus capacidades de crecimiento. La historia del SEP se ve marcada por esfuerzos individuales, o de pequeños colectivos, que creyeron que era su responsabilidad compartir los conocimientos adquiridos por ellos y ellas en el exterior y crear en la Universidad de Costa Rica fuentes de renovación generacional y de creación de conocimiento.

El posgrado, mayoritariamente constituido por programas de maestría académica, 
crece dinámicamente en la década de los 80 s y se consolida en el contexto de las unidades académicas base y colaboradoras, produciendo aún más presión sobre los fondos estatales de exiguo crecimiento. Al final de esta década se toma conciencia de que el posgrado en la Universidad de Costa Rica no puede crecer al ritmo que el país requiere, ni a la velocidad y con la calidad que la generación de nuevos profesores desean para consolidar la investigación. La Universidad de Costa Rica comienza a visualizar que la única forma de responder al país, con las opciones de posgrado que éste demanda, es a través de mecanismos alternos de generación de fondos.

Sirven de referentes Programa de Estudios de Posgrado en Ciencia Agrícolas y Recursos Naturales del CATIE [5] y la Maestría en Administración de Negocios, que inició la National University en 1983 [6].

Con base en una alianza estratégica entre la Universidad de Costa Rica (UCR) y National University, de San Diego, California, y a través de la creación de una fundación se logró que la Universidad de Costa Rica administrara y diera continuidad al programa [6]. En marzo de 1994, la Fundación FUNDEPOS ALMAMATER suscribió un convenio de cooperación recíproca con la Universidad de Costa Rica, mediante el cual se responsabilizó del desarrollo y la administración autofinanciada de los programas de Maestría en Administración de Negocios (M.B.A.) el cual sirve de inspiración para un programa propio de la Universidad a partir del 2003.

La posibilidad de crear programas con fuentes de financiamiento no estatal catapultó la creación de maestrías profesionales en la década de los 90s.

Nacen así distinciones entre los mecanismos de financiamiento para los programas de posgrado. Los programas tradicionales con financiamiento estatal fueron denominados regulares. Los programas nuevos, con fuentes de financiamiento alternativas, ya fuera por cobro de matrículas más elevadas, o por pago de organismos externos, se denominaron inicialmente autofinanciados, y luego de financiamiento complementario.

\section{PROGRAMAS DE POSGRADO DE FINANCIAMIENTO COMPLEMENTARIO}

Los programas de posgrado con financiamiento complementario perciben un pago por matrícula distinto respecto de los programas regulares, o reciben fondos externos provenientes de instituciones u organismos nacionales o internacionales, públicos o privados.

Rev. GUAL., Florianópolis, v. 4, n. 3, p.01-20, set/dez. 2011 
Estos fondos no ingresan a la cuenta única de la Universidad, sino que son administrados por los programas individualmente para garantizar su sostenibilidad. La Universidad de Costa Rica les aporta además, en mayor o menor grado, recursos adicionales de diversa índole (recursos humanos, infraestructura, gastos fijos, etc.), para facilitar su adecuado funcionamiento.

El Consejo Universitario, preocupado por la proliferación de este tipo de programas, y por la falta de normativa para su control, dicta en 1995 los Lineamientos para la Gestión de los Programas de Posgrado con Financiamiento Complementario [4]. Inicia dicha normativa dejando claro que el objetivo de estos programas no es la privatización:

"Estos programas deben ser congruentes con los principios, propósitos y funciones de la Universidad de Costa Rica como universidad pública y humanista, para contribuir al logro del bien común, promoción de la justicia social, la ética y el desarrollo integral de la sociedad." [4]

Continúa enfatizando la necesidad de un sano equilibrio entre pertinencia y sostenibilidad económica:

\footnotetext{
"Los Programas con financiamiento complementario deben buscar un sano equilibrio entre el impacto social directo e indirecto de sus actividades académicas y los recursos financieros que perciben. Su razón de ser es la acción académica y hacia esa meta deben dirigirse mediante una planificación y organización eficiente de sus actividades docentes y sus recursos propios y externos." [4]
}

Los Programas de posgrado con financiamiento complementario tienen una especial forma de gestión, derivada de su financiamiento.

Estos programas procuran cubrir sus costos operativos con los ingresos por cobro de matrícula. En la actualidad, año 2011, el costo de matrícula por ciclo para un programa regular asciende a $\mathbb{C} 138,000$ equivalente a US\$270 por una matrícula típica de 12 créditos mientras que un estudiante en un programa de posgrado de financiamiento complementario debería pagar por un costo del crédito específico que puede oscilar entre $\mathbb{C} 23,954$ y $\mathbb{C} 40,480$ (de acuerdo a los Lineamientos [4] de $\not 34,216+$ o - 30\%). Es decir, entre US\$ 47 y US\$ 79 por crédito, para un pago total por ciclo de 12 créditos entre US\$ 564 y US\$ 952. Un estudiante de un programa de financiamiento complementario podría tener que pagar el doble o hasta 3.5 veces lo que pagaría en un programa de posgrado regular. 
Además de cubrir los costos operativos propios de sus programas de posgrado, éstos están llamados por los Lineamientos a proporcionar una fuente de apoyo económico solidario al SEP como un todo. Se crea el Fondo Solidario (Fondo 170) y el Fondo de Becas (Fondo 082), destinados al mejoramiento integral de los posgrados que constituyen el Sistema de Estudios de Posgrado (SEP) y a apoyar a los estudiantes de bajos recursos. Estos son alimentados por un porcentaje de los ingresos de los programas de financiamiento complementario (10\%-15\%) y por el $50 \%$ de los excedentes anuales de los mismos. [4]

Con la figura de programas de financiamiento complementario, la Universidad delega en sus Programas de Posgrado la responsabilidad de generar los fondos para su sostenibilidad. Como se muestra en la Figura 2, la oferta de programas regulares es aprox. solo el $40 \%$ de la oferta académica de posgrados en la actualidad. De la figura se puede observar que las especialidades y las maestrías profesionales son los posgrados que mayoritariamente han tenido que asumir esta modalidad de financiamiento. Tanto es así que muchas veces se tiende a confundir, en el contexto universitario, los términos de grado profesional con la fuente de financiamiento complementario, lo cual no es del todo cierto.

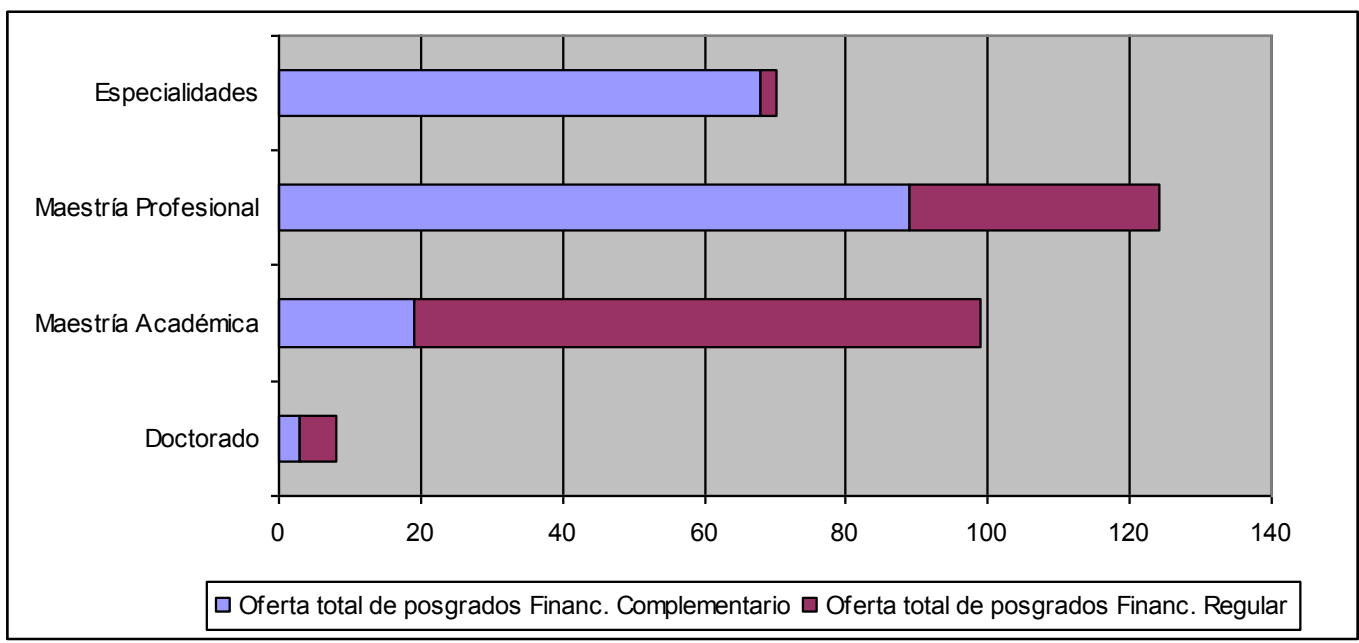

Figura 2 Cantidad de posgrados existentes en la Universidad de Costa Rica por grado académico y fuente de financiamiento (2010).

Es importante aclarar que existen posgrados de financiamiento complementario de alta demanda, lo cual permite que dichos programas puedan generar los fondos necesarios para su sostenibilidad. Este es el caso del Programa de Posgrado en Especialidades Médicas, con 53 especialidades y más de 800 estudiantes activos, o del caso del Programa de Posgrado en 
Administración de Empresas, con aproximadamente 180 estudiantes activos.

Existen una mayoría de casos de posgrados de financiamiento complementario de mediana o baja demanda, lo cual no permite que estos tengan una matrícula numerosa e ingresos suficientes para cubrir sus costos de operación. Es importante resaltar que para el 2010, 39 programas de posgrado cuentan con promociones inferiores a 20 estudiantes mientras que solo 19 contaron con promociones superiores a 20 estudiantes ${ }^{1}$.

Vale la pena resaltar la gran heterogeneidad en tamaño de los posgrados de financiamiento complementario. Unos, los grandes, tal vez 3 o 4 de ellos, pueden considerarse casi autosuficientes. Otros, la gran mayoría, son programas de muy escasos recursos, los cuales han de recurrir a fondos de sus unidades académicas y del SEP para cubrir el pago de profesores y la compra de materiales básicos para su subsistencia. Con sus ingresos propios difícilmente cubren su apoyo secretarial, y el pago de un porcentaje bajo de sus docentes.

Alarma la falta de coherencia entre la visión universitaria de que estos programas viven en la opulencia, y su realidad: la carestía de recursos con la cual deben existir. Preocupa además la percepción, también casi generalizada, de que estos programas no son de la misma calidad que los programas regulares. Es esta última percepción que motiva al análisis comparativo entre los diferentes tipos de posgrados que se presenta a continuación.

\section{ANÁLISIS COMPARATIVO DEL DESEMPEÑO Y DE LA GESTIÓN CON BASE EN EL INFORME DE LABORES 2010}

Durante el 2010, la Decanatura del SEP realizó un esfuerzo por automatizar el Informe de Labores que cada Programa de Posgrado debe entregarle como resultado de su gestión anual. Durante años, la información contenida en estos informes era de poca utilidad debido a que el SEP debe darle seguimiento a 67 Programas que imparten más de 280 posgrados distintos. La valiosa información contenida en dichos informes era rara vez consultada, y estudios comparativos entre programas requerían de la estandarización y digitación de la información, y por lo tanto, eran rara vez elaborados. En el 2010 los Programas de Posgrado fueron capacitados para completar su Informe de Labores a través de un instrumento basado en los indicadores de gestión del Manual de Acreditación de la Agencia Centroamericana de

\footnotetext{
${ }^{1}$ Información generada por el SEP y comunicada a la Oficina de Administración Financiera con fecha 26 de enero de 2011 (SEP-232-2011).
}

Rev. GUAL., Florianópolis, v. 4, n. 3, p.01-20, set/dez. 2011 
Acreditación de Postgrado, ACAP [1], y elaborado por el SEP utilizando una plataforma de software libre para realizar encuestas en línea llamada Lime Survey [7].

La Figura 3 muestra la cantidad de posgrados que respondieron al Informe de Labores por grado académico y fuente de financiamiento. El Cuadro 1 muestra la tasa de respuesta al Informe de Labores por grado académico y fuente de financiamiento. Nótese en este cuadro que los posgrados de financiamiento complementario presentan una tasa más alta de respuesta al Informe de Labores que los programas regulares, para todos los grados académicos salvo Especialidades. Esto se debe a que el instrumento no es muy adecuado para el Programa de Especialidades Médicas, que involucra 53 especialidades, y no fue invitado a completar el instrumento.

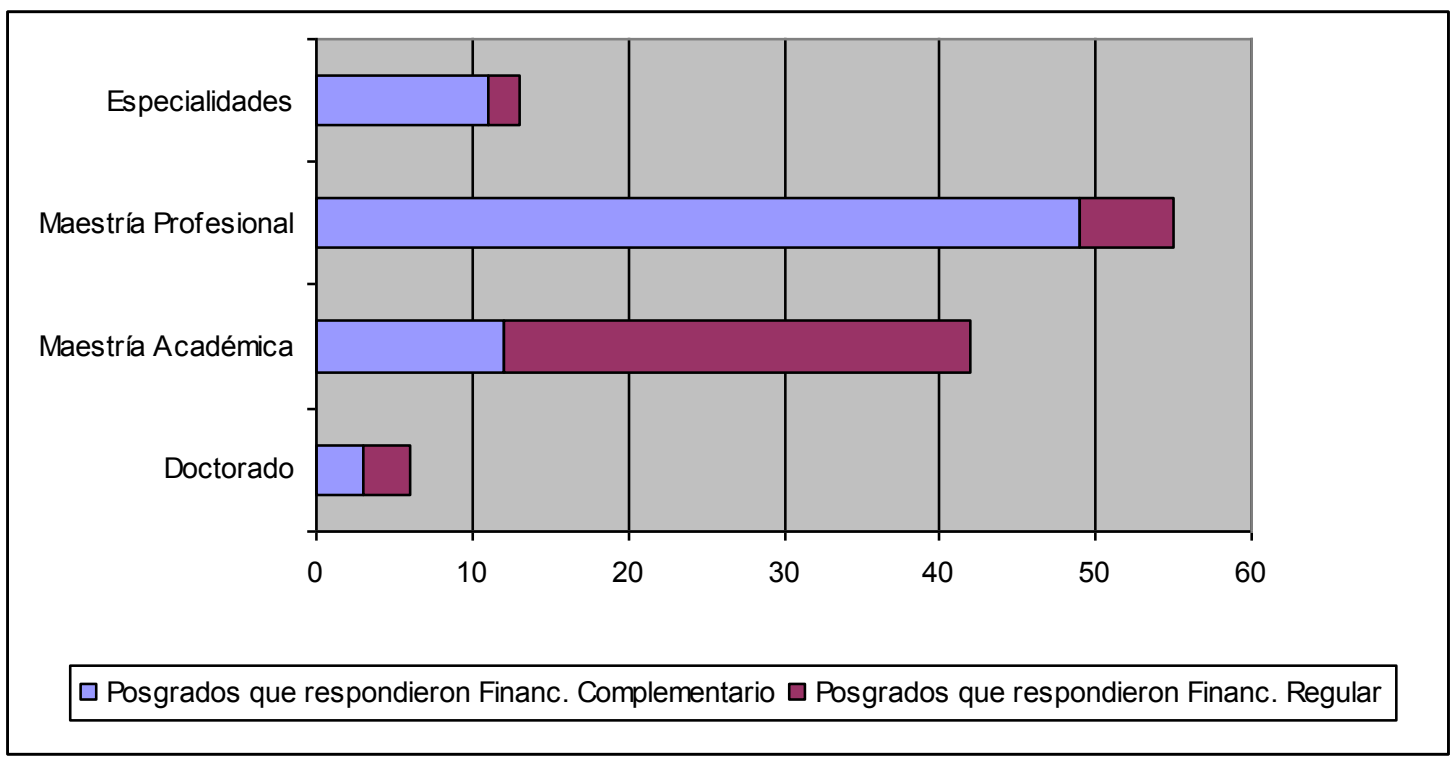

Figura 3 Cantidad de posgrados que respondieron al Informe de Labores por grado académico y fuente de financiamiento (2010).

\section{Cuadro 1}

Tasa de respuesta al Informe de Labores 2010 por tipo de financiamiento.

\begin{tabular}{|ccc|}
\hline Tasa de respuesta & $\begin{array}{c}\text { Financiamiento } \\
\text { Complementario }\end{array}$ & $\begin{array}{c}\text { Financiamiento } \\
\text { Regular }\end{array}$ \\
Doctorado & $100 \%$ & $60 \%$ \\
Maestría Académica & $63 \%$ & $38 \%$ \\
Maestría Profesional & $55 \%$ & $17 \%$ \\
Especialidades & $16 \%$ & $100 \%$ \\
\hline
\end{tabular}

Rev. GUAL., Florianópolis, v. 4, n. 3, p.01-20, set/dez. 2011 
Los indicadores de desempeño y de la gestión de los posgrados presentados a continuación están basados en datos auto-reportados, y solo de los posgrados que respondieron al Informe de Labores.

\section{COMPARACIÓN ENTRE POSGRADOS POR TIPO DE FINANCIAMIENTO}

A continuación se listan una serie importante de criterios de comparación entre los posgrados se diferentes tipos de financiamiento. Por limitaciones de espacio, se reporta los criterios en los cuales los posgrados presentan diferencias únicamente.

\section{- Versatilidad del plan}

Los posgrados de financiamiento complementario dependen en mayor medida de contar con un número adecuado de estudiantes que los posgrados regulares. De hecho, la sostenibilidad de estos dependen de los ingresos por pago de matrícula, lo cual hace que la modalidad con la cual son impartidos se deba ajustar a las características de la población meta. Esto se refleja no solo en la diversidad de medios para su divulgación (presentado más adelante) sino que ha causado que se utilice una diversidad mucho mayor de diseños curriculares en adición al semestral, el cual es tradicional en nuestra Universidad. Existen un número creciente de posgrados de financiamiento complementario cuatrimestrales y trimestrales, como se observa en la Figura 4 para lograr aprovechar todo el año lectivo.

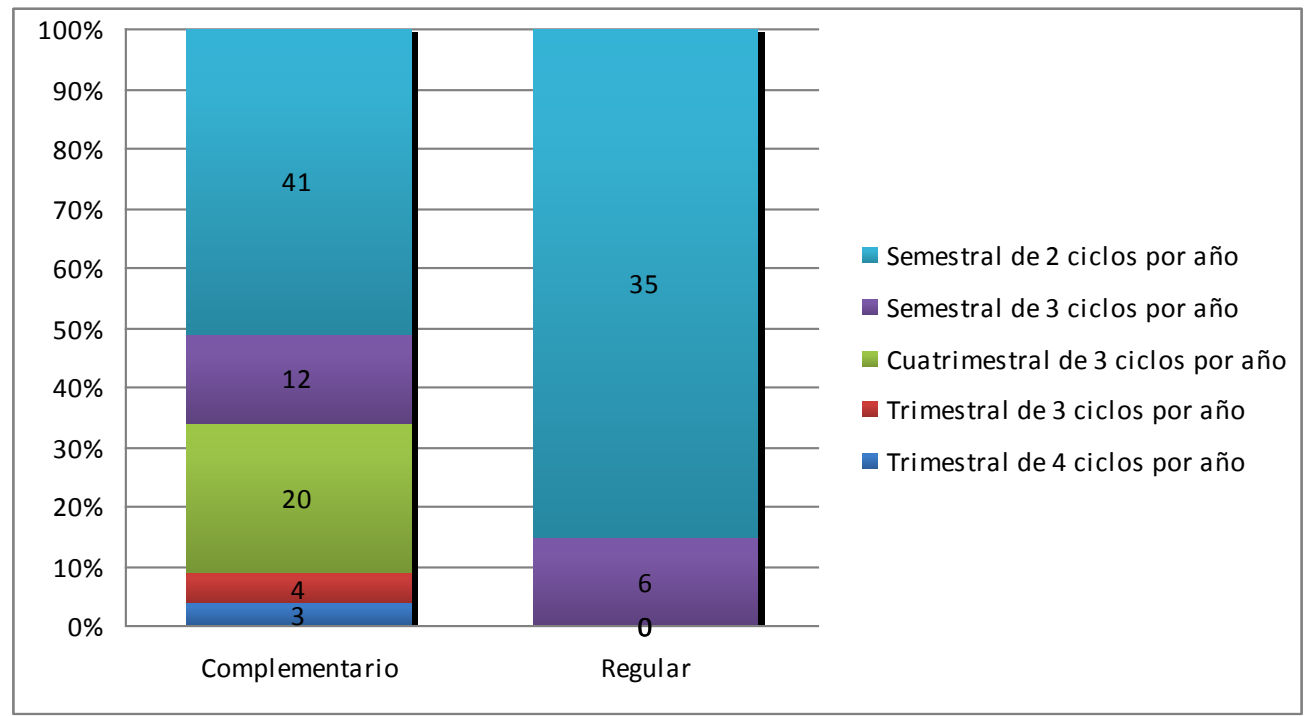

Figura 4 Cantidad de posgrados por modalidad de ciclo por tipo de financiamiento (2010). 


\section{- Internacionalización de la población estudiantil}

Otro indicador que también presenta diferencias significativas entre los dos tipos de posgrados es la tasa de internacionalización de la población estudiantil.

Los programas regulares parecen ser un poco más atractivos que los de financiamiento complementario para los centroamericanos. Esta diferencia se debe, posiblemente, a que son los posgrados más consolidados en el tiempo, y han logrado más becarios del Agencia Alemana de Cooperación DAAD.

Cuadro 2 Nacionalidad de los estudiantes por tipo de financiamiento (2010)

\begin{tabular}{|l|ccc|ccc|}
\hline \multirow{2}{*}{ Procedencia } & \multicolumn{3}{|c|}{ Complementario } & \multicolumn{3}{c|}{ Regular } \\
\cline { 2 - 7 } & $\begin{array}{c}\text { Total de } \\
\text { estudiantes }\end{array}$ & Porcentaje & $\begin{array}{c}\text { Promedio por } \\
\text { Posgrado 1/ }\end{array}$ & $\begin{array}{c}\text { Total de } \\
\text { estudiantes }\end{array}$ & Porcentaje $\begin{array}{c}\text { Promedio por } \\
\text { Posgrado 2/ }\end{array}$ \\
\hline Costa Rica & 908 & 95.08 & 28.38 & 274 & 85.09 & 19.57 \\
Resto de Centro América & 10 & 1.05 & 0.31 & 19 & 5.90 & 1.36 \\
Otros países Latinoamérica & 25 & 2.62 & 0.78 & 7 & 2.17 & 0.50 \\
Estados Unidos y Canadá & 9 & 0.94 & 0.28 & 3 & 0.93 & 0.21 \\
Europa & 2 & 0.21 & 0.06 & 3 & 0.93 & 0.21 \\
África, Asia, Oceanía & 1 & 0.10 & 0.03 & 0 & 0.00 & 0.00 \\
Sin información & 0 & 0.00 & 0.00 & 16 & 4.97 & 1.14 \\
\hline Total & 955 & 100.00 & 29.84 & 322 & 100.00 & 23.00 \\
\hline $\begin{array}{l}\text { 1/ Estudiantes admitidos / 32 Posgrados que admitieron estudiantes en el 2010 } \\
\text { 2/ Estudiantes admitidos / 14 Posgrados que admitieron estudiantes en el 2010 }\end{array}$ \\
\hline
\end{tabular}

\section{- Fuentes de financiamiento de la población estudiantil}

Los estudiantes de posgrado son en su mayoría profesionales que trabajan y estudian a la vez. Ellos típicamente cubren los costos de matrícula de sus estudios de posgrado. Es interesante resaltar que un porcentaje similar de ellos tienen algún tipo de beca para ambas modalidades de posgrados. Sin embargo, la fuente principal de becas, o exoneración parcial o total de matrícula corresponde en los posgrados de financiamiento complementario a los mismos Programas (los Lineamientos establecen la obligatoriedad de destinar el $20 \%$ de ingresos a becas, con el fin de preservar la filosofía de universidad pública, y garantizar la accesibilidad a los estudios [4]). 


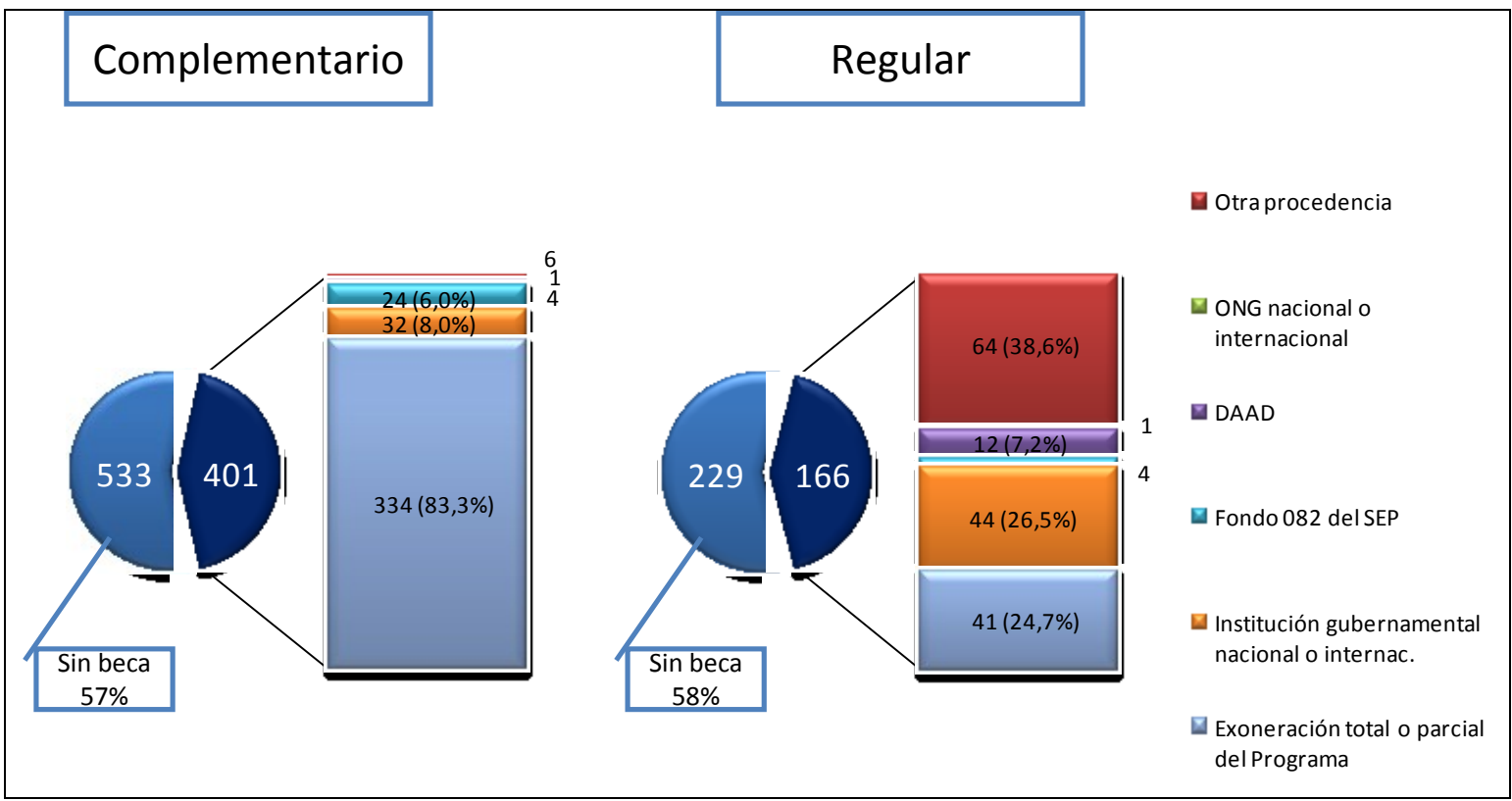

Figura 5 Fuentes de exoneración y becas estudiantiles por tipo de posgrado (2010).

A continuación reportamos diferencias en la gestión de los programas en sí.

\section{GESTIÓN DE LOS PROGRAMAS DE POSGRADO}

Los posgrados son administrados por Programas de Posgrado, lo cuales están presididos por una Comisión. Los Programas de Posgrado pueden impartir diferentes posgrados. Por ejemplo, el Programa de Posgrado en Historia es responsable del Doctorado en Historia y de la Maestría Académica en Historia; en este caso, ambos de tipo regular. El Programa de Posgrado en Computación e Informática alberga la Maestría Profesional y la Maestría Académica con el mismo nombre; ambos posgrados de financiamiento complementario.

Existen Programas de Posgrados denominados mixtos ya que administran posgrados regulares y posgrados de financiamiento complementario; como por ejemplo, el Programa de Posgrado en Ciencias Políticas, responsable de la Maestría Académica en Ciencias Políticas con énfasis en Políticas Públicas y Gobernabilidad que es un posgrado regular y la Maestría Profesional en Ciencias Políticas con énfasis en Gerencia Política, que es de financiamiento complementario. El Cuadro 3 muestra la distribución de los Programas de Posgrado que llenaron el Informe de Labores y del cual obtenemos las estadísticas para tipificar las diferencias y similitudes entre los distintos tipos de gestión. 
Cuadro 3 Número de Programas de Posgrado por tipo de financiamiento para los posgrados que albergan (2010)

\begin{tabular}{|c|c|c|}
\hline Financiamiento & Programas & Porcentaje \\
\hline Complementario & 28 & $54,9 \%$ \\
\hline Regular & 15 & $29,4 \%$ \\
\hline Mixto & 8 & $15,7 \%$ \\
\hline Total & $\mathbf{5 1}$ & $\mathbf{1 0 0 , 0} \%$ \\
\hline
\end{tabular}

A continuación comparamos el desempeño en términos de su gestión, para programas regulares, de financiamiento complementario, y mixtos.

\section{- Mecanismos de divulgación}

Como se planteó anteriormente, los posgrados de financiamiento complementario dependen en mayor medida de contar con un número adecuado de estudiantes que los posgrados regulares, y por lo tanto, los programas que los administran realizan más divulgación, lo cual es evidente en la Figura 6. Resalta el hecho que Programas de Posgrado mixtos que administran posgrados de ambas modalidades, son aún más agresivos para divulgar sus posgrados. Posiblemente tienen poblaciones más heterogéneas, o simplemente son más concientes de la necesidad de segmentar su población meta (y por eso son mixtos), y de divulgar sus planes de estudio.

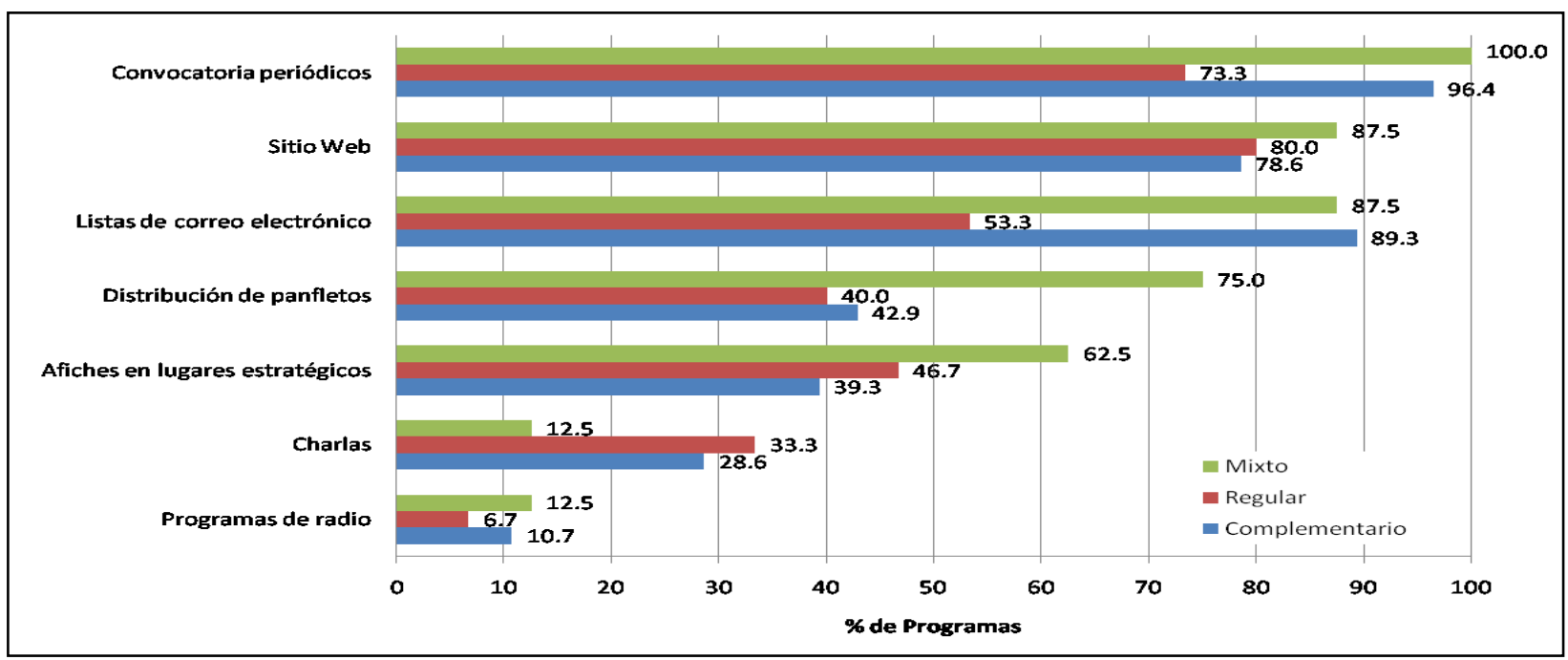

Figura 6 Medios de divulgación utilizados por tipo de programa (2010). 


\section{- Capacitación continua del personal docente}

La Figura 7 muestra claramente como los docentes en los programas de posgrado mixtos son, en promedio, mucho más proactivos en sus procesos de capacitación continua y desarrollo profesional que los de financiamiento complementario y los regulares, en todas y cada una de las categorías reportadas en el Informe de Labores 2010. Más sorprendente aún es el hecho que los docentes de los programas de financiamiento complementario, los cuales tienen en general menor vínculo laboral en promedio para con la Universidad, reporten mayor actividad en su autoformación que los programas de posgrado regulares.

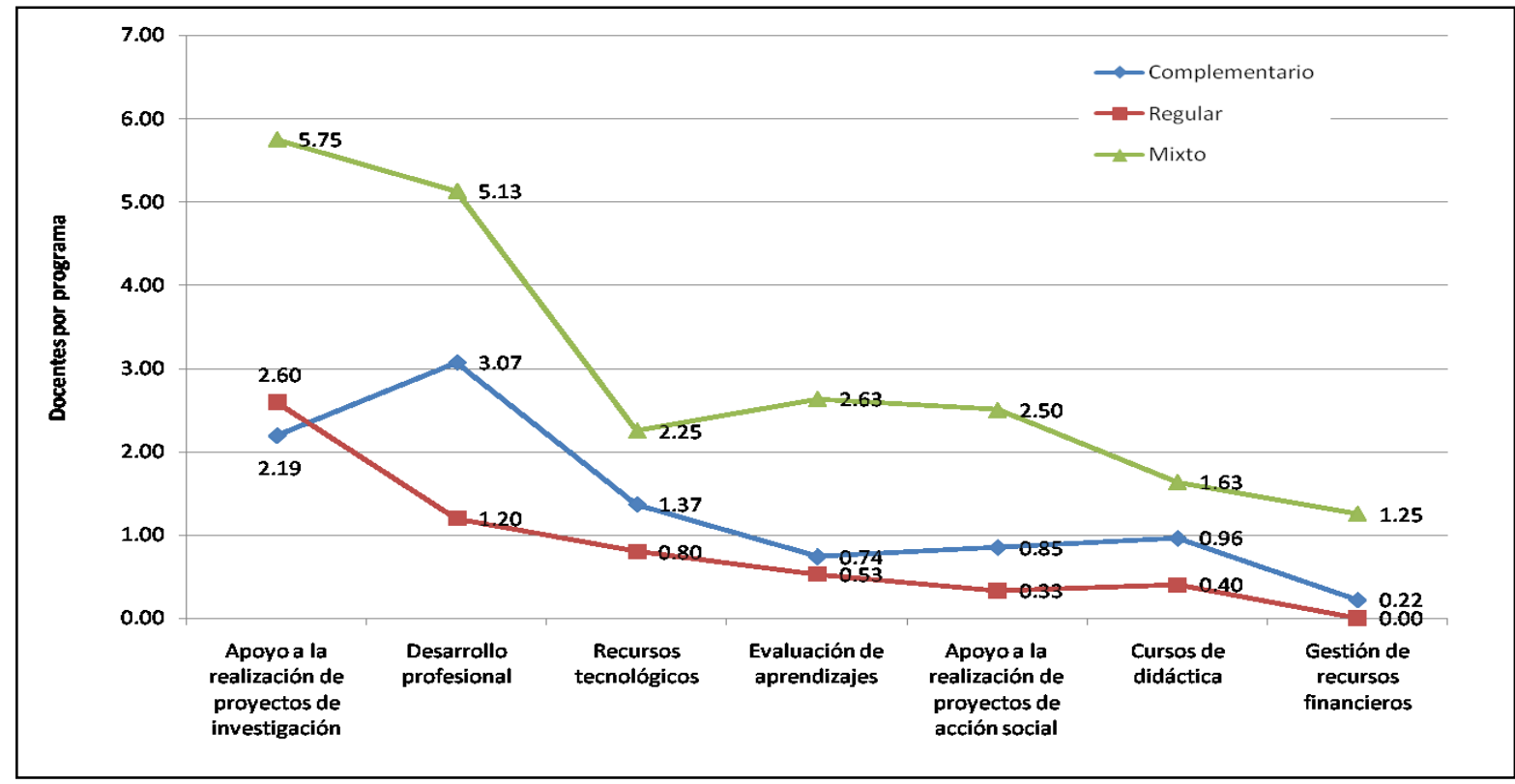

Figura 7 Participación docente en actividades de capacitación: promedio de docentes por programa (2010).

Esto puede deberse en parte a que los profesores de los programas regulares tienden a ser veteranos, mientras que en los programas de financiamiento complementario son más jóvenes típicamente.

Vale resaltar el hecho de que en la última categoría, "Gestión de recursos financieros", los docentes de los programas regulares no participan del todo. Esto no es necesariamente beneficioso pues la formación para la consecución de fondos externos viene acompañada de la posibilidad del aprovechamiento de oportunidades para realizar proyectos de investigación de mayor envergadura.

Es interesante ligar esta variable con los resultados reportados de divulgación de la 
investigación que citamos a continuación.

\section{- Divulgación de la investigación}

Las estadísticas auto-reportadas por los Programas de Posgrado sobre la divulgación de la investigación realizada se resumen en el Cuadro 3 (investigación hecha por los docentes), y en el Cuadro 4 (investigación que involucra estudiantes).

Nuevamente llama la atención que, porcentualmente, los Programas de Posgrado mixtos revelan un mayor porcentaje que los otros dos tipos de programas en publicaciones durante el 2010. Aún más, estos programas presentan cifras mayores en los indicadores "Promedio por programas totales" y "Promedio por programas con publicaciones", lo cual se encuentra resaltado en azul e itálica en el Cuadro 3.

Cuadro 3 Programas con publicaciones realizadas por docentes (2010)

\begin{tabular}{c|ccc}
\multirow{2}{*}{ Publicaciones } & \multicolumn{3}{|c}{ Financiamiento } \\
\cline { 2 - 4 } & Complementario & Regular & Mixtos \\
\hline 1 5 & 6 & 5 & 1 \\
6 a 10 & 13 & 4 & 1 \\
11 a 15 & 4 & 4 & 3 \\
16 y más & 4 & 1 & 1 \\
\hline Programas totales & 0 & 1 & 2 \\
\hline Programas con publicaciones & 27 & 15 & 8 \\
\% Programas con publicaciones & 21 & 10 & 7 \\
Publicaciones totales & $77,8 \%$ & $66,7 \%$ & $87,5 \%$ \\
Promedio por programas totales & 116 & 135 & 162 \\
Promedio por programas con & 4,30 & 9,00 & 20,25 \\
publicaciones & 5,52 & 13,50 & 23,14 \\
\hline
\end{tabular}

Similarmente, en el Cuadro 4 se resaltan en azul e itálica los indicadores de los Programas de Posgrado mixtos, los cuales revelan un mayor porcentaje que los otros dos tipos de programas en publicaciones en las cuales se involucra al menos un estudiante del programa durante el 2010. Estos programas presentan cifras mayores en los indicadores "Promedio por programas totales" y "Promedio por programas con publicaciones". 
Cuadro 4 Programas con publicaciones realizadas que incluyen estudiantes (2010)

\begin{tabular}{c|ccc}
\multirow{2}{*}{ Publicaciones } & \multicolumn{3}{|c}{ Financiamiento } \\
\cline { 2 - 4 } & Complementario & Regular & Mixtos \\
\hline 0 & 19 & 12 & 4 \\
1 a 5 & 7 & 2 & 3 \\
6 10 & 1 & 1 & 0 \\
11 y más & 0 & 1 & 1 \\
\hline Programas totales & 27 & 15 & 8 \\
Programas con publicaciones & 8 & 4 & 4 \\
\% Programas con publicaciones & $29,6 \%$ & $26,7 \%$ & $50,0 \%$ \\
Publicaciones totales & 15 & 14 & 44 \\
Promedio por programas totales & 0,56 & 0,93 & 5,50 \\
Promedio por programas con & 1,88 & 3,50 & 11,00 \\
publicaciones & & & \\
\hline
\end{tabular}

La diversidad de tipos de publicaciones que se dieron durante el 2010 por los docentes y algunos de los estudiantes de los programas de posgrado se refleja en a Figura 8, la cual desglosa los promedios por tipo de publicación y por tipo de programa.

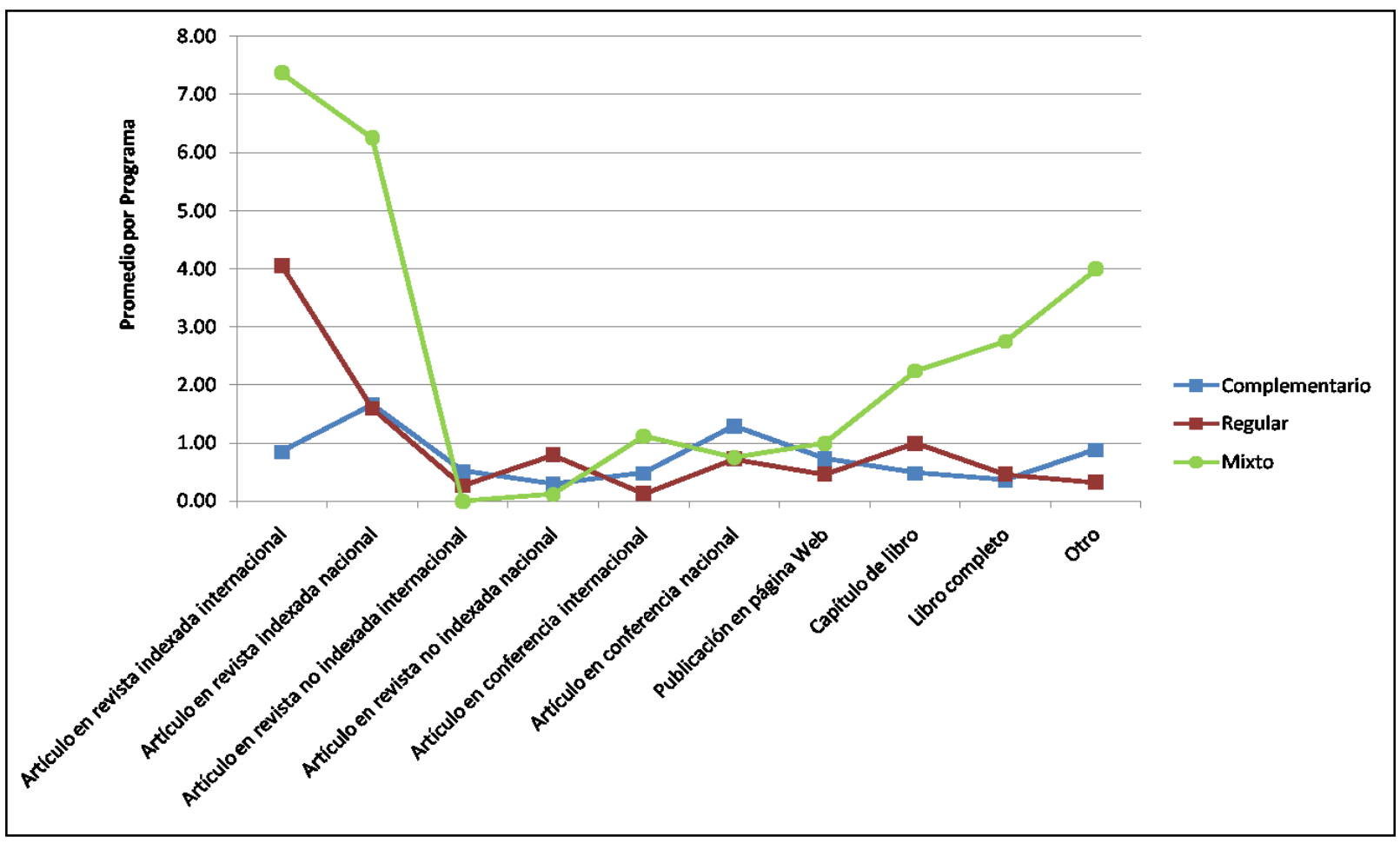

Figura 8 Desglose por tipos de publicaciones: promedios por tipo de programa (2010). 
Es importante realzar que el tipo de publicación con mayor prevalencia es la de "Artículos en revista indexada internacional", y en segundo lugar "Artículos en revista indexada nacional", seguidos por "Capítulos de libro" y "Libros completos", lo cual pone en evidencia la calidad de nuestros docentes, y de los programas de posgrado en general. Nuevamente, los programas de posgrado mixtos destacan por encima de las otras dos modalidades, en estas cuatro categorías. En ese caso, los programas de posgrado regulares sí presentan un promedio mayor que los de financiamiento complementario en la categoría "Artículos en revista indexada internacional".

\section{CONCLUSIONES}

En el transcurso de estas líneas se ha tratado de dar una panorámica de la Educación Superior Universitaria costarricense y su evolución histórica producto de la presión por responder a las necesidades del país en forma pertinente y oportuna pero en un mundo de crecientes limitaciones presupuestarias a partir de la crisis de los años ochentas. En particular se trata de documentar el nacimiento de una nueva figura de gestión universitaria en el seno del Sistema de Estudios de Posgrado (SEP) denominada posgrado de financiamiento complementario; figura vista por algunos como la solución para obtener fuentes alternativas de financiamiento para posibilitar el crecimiento dentro del seno de una universidad pública, pero vista por otros, como la "privatización" o la "creación de una universidad paralela" en el seno de la Universidad de Costa Rica.

Se ha hecho un esfuerzo por presentar muchos de los mitos que han llegado a formar parte en el imaginario de un sector de la Universidad y proveer evidencia empírica sobre las realidades que los diferentes tipos de gestión en el seno del SEP. Se evidencia como una realidad que la figura de financiamiento complementario se ha ido expandiendo en la UCR y que la oferta de programas regulares es aproximadamente solo el $40 \%$ de la oferta académica de posgrados en la actualidad. También se afirma la creencia que las especialidades y las maestrías profesionales son los posgrados que mayoritariamente han tenido que asumir esta modalidad de financiamiento.

Alarma la falta de coherencia entre la visión universitaria de que estos programas viven en la opulencia, y su realidad: la carestía de recursos con la cual deben existir. Preocupa además la percepción, también casi generalizada, de que estos programas no son de 
la misma calidad que los programas regulares. El análisis comparativo entre los diferentes tipos de posgrados según los datos auto-reportados en el Informe de Labores 2010, evidencian paridad en muchísimos de los criterios de calidad reflejados por el Informe de Labores el cual se inspira en el Manual de Acreditación del ACAP [1]. Por aspectos de espacio, en este artículo no reportamos las similitudes, sino solo reportamos las diferencias entre los tipos de programas.

Los programas regulares pareciera que viven en una zona de mayor confort que los adormece en algunos aspectos importantes como: la adecuación de su oferta académica a las necesidades de la población profesional de utilizar la totalidad del año en su formación, la diversidad de medios de divulgación existentes para la atracción de los mejores candidatos, la necesidad de capacitación continua de sus docentes, y más sorprendentemente, la necesidad de divulgar sus resultados de investigación. Los posgrados de financiamiento complementario, por otro lado, han sido más débiles en buscar atraer estudiantes centroamericanos para enriquecer sus experiencias en el aula y tener un mayor impacto regional.

El descubrimiento más interesante de la investigación fue la supremacía en la gestión de los programas de posgrado mixtos, los cuales administran programas regulares y de financiamiento complementario, en su seno, sobre los programas homogéneos. Estos programas muestran: mayor agresividad en el uso de medios de divulgación para la atracción de la población estudiantil, mayor conciencia de parte de sus docentes para involucrarse en actividades de formación continua y lo más importante, mayor efectividad para divulgar sus resultados de investigación e involucrar a los estudiantes en esta divulgación.

El Sistema de Estudios de Posgrado ve con muy buenos ojos estos resultados, los cuales sirven para desmitificar muchas de las percepciones universitarias de que los programas de financiamiento complementario son reductos universitarios para la venta de servicios, que carecen de motivaciones académicas. Estudios longitudinales y a mayor profundidad son necesarios para obtener conclusiones más certeras, sin embargo, el análisis comparativo del Informe de Labores 2010 nos llama a la reflexión y nos permite conocer más la gestión de nuestros programas de posgrado. 


\section{AGRADECIMIENTOS}

Se agradece a la Unidad de Evaluación y Gestión de la Calidad del Sistema de Estudios de Posgrado por todo el esfuerzo invertido en el diseño, ejecución y presentación de resultados del Informe de Labores 2010, y en especial a la M.Sc. Lorena Kikut, funcionaria encargada de este proceso.

\section{REFERENCIAS}

[1] Agencia Centroamericana de Acreditación de Postgrado, ACAP, Manual de acreditación de ACAP,

http://acap.csuca.org/index.php?option $=$ com_remository\&Itemid $=109 \&$ func $=$ fileinfo $\& \mathrm{id}=9$

[2] Constitución Política de la República de Costa Rica

http://www.tramites.go.cr/manual/espanol/legislacion/ConstitucionPolitica.pdf

[3] Consejo Nacional de Rectores. Normativa sobre el Financiamiento de la Educación Superior Universitaria Estatal de Costa Rica / Consejo Nacional de Rectores, Oficina de Planificación de la Educación Superior.-San José Costa Rica: CONARE OPES publicaciones 2003. http://proyectos.conare.ac.cr/doctextcomp/opes/2003/OPES17_2003.pdf

[4] Consejo Universitario. Lineamientos para la Gestión de los Programas de Posgrado con Financiamiento Complementario. (Reforma integral aprobada en sesión 4922-02, 14-10-04. Publicada en Gaceta Universitaria 33-2004, 19-11-04. Rigen

a partir del presupuesto del 2005),

http://www.cu.ucr.ac.cr/normativ/posgrado_financiamiento_compl.pdf

[5] Cordero, Alvaro. Informe del Programa de Estudios de Posgrado en Ciencia Agrícolas y Recursos Naturales del CATIE http://orton.catie.ac.cr/repdoc/A6600E/A6600E.PDF

[6] FUNDEPOS. Fundación de Estudios de Posgrado e Investigación en Ciencias Económicas FUNDEPOS ALMAMATER-Perfil, http://www.unire.or.cr/universidades/fundepos.htm

[7] Lime Survey. http://www.limesurvey.org/es

[8] Pérez, María. Unversidad de Costa Rica: Premisas, polémicas y propuestas actuales en educación. Revista educación 27(1): 95-113, 2003. http://redalyc.uaemex.mx/pdf/440/44027109.pdf

[9] PNUD, Informe 2010 del Programa de las Naciones Unidas para el Desarrollo. http://hdr.undp.org/en/reports/ 


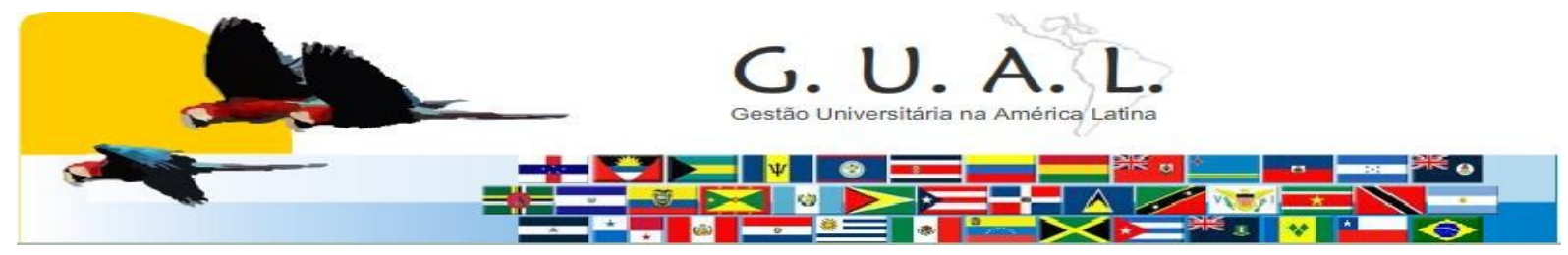

ISSN 1983-4535

\title{
GRADUATE PROGRAMS WITH COMPLEMENTARY FINANCING: MYTHS AND REALITIES
}

\begin{abstract}
Myths and realities of what has been called the privatization of education in the context of a public university are presented. Graduate programs fully funded with public funds, called regular programs, and graduate programs with complementary funding are compared. The study is based on self-reported data of the 2010 Annual Report. The report template includes indicators from the Manual for Accreditation of the Central American Accreditation Agency, ACAP, and was developed by Graduate Studies System the University of Costa Rica using a free software platform for online surveys. Differences between regular programs, complementary funding programs, and mixed financing programs are examined, highlighting the benefits of the latter type of management.
\end{abstract}

Keywords: University management. Postgraduate programas. Funding. 